\title{
Active Targeting Liposomes: Promising Approach for Tumor-Targeted Therapy
}

\author{
Xiaoyi Wang ${ }^{1}$ and Weiyue $\mathrm{Lu}^{1-3^{*}}$ \\ ${ }^{1}$ Department of Pharmaceutics, School of Pharmacy, Fudan University \& Key Laboratory of Smart Drug Delivery (Fudan University), Ministry of Education, Shanghai \\ 201203, PR China \\ ${ }^{2}$ State Key Laboratory of Medical Neurobiology, The Collaborative Innovation Center for Brain Science, Fudan University, Shanghai 200032, PR China \\ ${ }^{3}$ State Key Laboratory of Molecular Engineering of Polymers, Fudan University, Shanghai 200433, PR China
}

A good bioavailability is a prerequisite for the function of drugs and thus is considered as an important standard to evaluate the quality of drug products. However, with the emerge of precision medicine [1], a good bioavailability becomes far less enough for assessment of drugs intended to be delivered into specific tissues, cells, or even organelles, especially for drugs with serious side effects such as anticancer drugs. The purpose of this commentary is to discuss the use of drug delivery systems, especially liposomes, to solve the problem of non-specific distribution of free anticancer drugs through passive or active tumor targeting.

Liposomes are artificially prepared vesicles composed of a biocompatible lipid bilayer and/or a concentric series of multiple bilayers that enclose a central aqueous compartment. Agents can be encapsulated in the aqueous compartment or intercalated in the bilayer membrane. The protective and solubilizing effect of liposomes can significantly improve the solubility, stability and blood circulation time of drugs. For example, the circulation time of sphingomyelin and cholesterol-based vincristine liposomes Marqibo is 6.6 hours (1.36 hours for free vincristine) $[2,3]$ and that of PEGylated doxorubicin liposomes Doxil ${ }^{\circ}$ can be as long as more than 350 hours [4]. Besides, due to the enhanced permeability and retention effect (EPR), liposomes can be passively targeted to tumors, allowing the loaded drugs more available to tumor cells thus further enhancing anticancer efficiency of drugs. Furthermore, modifiability of liposomes make the more active and precise targeting possible. Owing to all of these advantages, liposomes become the most successful drug delivery system with more than 12 drugs in routine clinical use, most of them are for cancer treatment, such as paclitaxel liposome for injection, doxorubicin liposomes (Doxil', Caelyx, Myocet, Doxorubicin Hydrochloride Liposome Injection), vincristine liposomes (Marqibo'), irinotecan liposomes (Onivyde), daunomycin liposomes (DaunoXome"), cytarabine liposomes (DepoCyt $)$, mifamurtide liposomes (Mepact ${ }^{\circ}$ ), etc.

At the same time, many more passive as well as active targeting liposomal products have progressed into clinical trials, e.g. thermo sensitive doxorubicin liposome Thermodox, irinotecan liposomes (LE-SN38, CPX-1 ${ }^{\circ}$ and $\mathrm{MM}-398^{\circ}$ ), tumor endothelial cells targeting paclitaxel cationic liposomes EndoTAG-1, cisplatin liposomes (Lipoplatin', SPI- $007^{\circ}$ and LiPlaCis'), and vinorelbine liposome Alocrest, etc. Among which, MM- $398^{\circ}$ has completed its Phase III study for the treatment of metastatic pancreatic cancer this year while the Phase III studies of Thermodox and Lipoplatin are recruiting. Besides, it should be pointed out that there are also several ligandtargeted liposomes are in different phases of clinical trials. As reviewed by Van der Meel R et al. [5], oxaliplatin liposome MBP-426 and tumor suppressor protein loading liposome SGT-53 are both designed to target transferrin receptor on tumor cells via the ligand transferrin and single-chain antibody fragment (scFv) respectively. Doxorubicin liposomes MM-302, Anti-EGFR ILs-DOX, 2B3-101 can actively target human epidermal growth factor receptor-2 (HER2), epidermal growth factor receptor (EGFR) and glutathione transporters on blood-brain barrier (BBB) via coupling of scFv antibody fragments, Fab' fragments of the anti-EGFR mAb cetuximab and glutathione (GSH) respectively. In addition, there are another two liposomes (MCC-465 with the conjugation of $\mathrm{F}\left(\mathrm{ab}^{\prime}\right) 2$ of the human GAH antibody and LipovaxinMM modified with melanoma antigens) are undergoing clinical trials as well. Most of formulations presented excellent targeting effect in preclinical studies and desirable response rate in clinical trials. For example, anti-EGFR ILs-DOX showed superior cancer cell association and internalization than ligand-lacking control formulations in vitro and significantly inhibited tumor growth in EGFR-overexpressing tumor xenograft models. Besides, it can even overcome multidrug resistance in drug resistant tumor xenograft model. In a phase I study of anti-EGFR ILs-DOX, among 26 patients with EGFR-overexpressing advanced solid tumors, one patient showed complete response, one partial response and ten patients had stable disease lasting 2-12 months. Because of encouraging results like this, many of ligand-targeted liposomes are recommended for further studies. However, as most of the ligand-targeted liposomes use proteins or antibodies as ligands, there are many potential problems for these formulations as this kind of ligands is easily to degrade in blood circulation and formulations will need strict storage conditions as well.

Our lab has done a lot of works on ligand-targeted liposomes as well. The difference is that we replace proteins and antibodies with stable peptides or small molecular compounds because these ligands show better stability while the targeting effects remain desirable. As the main goal of our research is to treat glioma, we modified liposomes with one or more peptides/small molecules which can target BBB or blood-brain tumor barrier (BBTB) and glioma cells. Among tested ligands, the PEGylated liposomes with dual peptide ligands [6], ${ }^{\mathrm{D}} \mathrm{CDX}$ and cyclic RGD (cRGD), show most promising prospect. ${ }^{\mathrm{D}} \mathrm{CDX}$ is a D-peptide ligand of nicotine acetylcholine receptors (nAChRs) on the $\mathrm{BBB}$ [7], and cRGD is a ligand of integrin highly expressed on the BBTB and glioma cells [8]. Both peptides are proved to stable in blood. Through the modification, liposomes can actively target both brain capillary endothelial cells and tumor neogenetic vessel cells, effectively traversing BBB and BBTB monolayers and targeting glioma cells. As a

*Corresponding author: Weiyue Lu, Department of Pharmaceutics, School of Pharmacy, Fudan University \& Key Laboratory of Smart Drug Delivery (Fudan University), Ministry of Education, Shanghai 201203, PR China, Tel: +862151980090; E-mail: wylu@shmu.edu.cn

Received November 24, 2015; Accepted December 18, 2015; Published December 25, 2015

Citation: Wang X, Lu W (2016) Active Targeting Liposomes: Promising Approach for Tumor-Targeted Therapy. J Bioequiv Availab 8: 013-014. doi:10.4172/ jbb. 1000260

Copyright: @ 2016 Wang X, et al. This is an open-access article distributed under the terms of the Creative Commons Attribution License, which permits unrestricted use, distribution, and reproduction in any medium, provided the original author and source are credited. 
Citation: Wang X, Lu W (2016) Active Targeting Liposomes: Promising Approach for Tumor-Targeted Therapy. J Bioequiv Availab 8: $013-014$. doi:10.4172/jbb.1000260

result, drugs encapsulated in dual ligand liposomes present better antiglioma effect with prolonged median survival of nude mice bearing glioma compared with free drug and non-dual ligands liposomes $(36.5$ days for dual ligand liposomes, 28 days for free drug and 29 days for non-dual ligands liposomes).

Except for our researches, studies by other groups also confirmed the effectiveness of peptide modification for tumor-targeted liposomal drug delivery. Reported formulations include multifunctional tandem peptide R8-c(RGD) modified liposomes [9], tumorpenetrating peptide (RGERPPR) attached liposomes [10], angiopep-2 and tLyP-1 dual ligand liposomes [11], etc. We believe that with researches going on, the benefits we can get from peptide modified liposomes will be greater.

All the encouraging results of liposome researches confirm that tumor active targeting liposomes as a kind of formulation with great possibility of translating into clinical applications, bring some hope for the treatment of cancer. However, as our knowledge of liposomes, tumor biology and interactions of liposomes in the body is still limited, to advance the applicability of ligand-targeted liposomes, further studies on in vitro/in vivo characterization of liposomes, receptor expression levels on various cancer cells and ligand-receptor reactions are needed [5].

\section{Acknowledgments}

This work was supported by National Basic Research Program of China (973 Program, 2013CB932500), National Natural Science Foundation of China (81273458 and 81473149) and National Science \& Technology Major Project (2012ZX09304004).

\section{References}

1. Jang SH, Yan ZG, Lazor JA (2015) Therapeutic Drug Monitoring: A Patient Management Tool for Precision Medicine. Clin Pharmacol Ther.

2. Krishna R, Webb MS, St Onge G, Mayer LD (2001) Liposomal and nonliposomal drug pharmacokinetics after administration of liposome-encapsulated vincristine and their contribution to drug tissue distribution properties. J Pharmacol Exp Ther 298: 1206-1212.

3. Davis T, Farag SS (2013) Treating relapsed or refractory Philadelphia chromosome-negative acute lymphoblastic leukemia: liposome-encapsulated vincristine. Int J Nanomedicine 8: 3479-3488.

4. Barenholz $Y$ (2012) Doxil(R)-the first FDA-approved nano-drug: lessons learned. J Control Release 160: 117-134.

5. van der Meel R, Vehmeijer LJ, Kok RJ, Storm G, van Gaal EV (2013) Ligandtargeted particulate nanomedicines undergoing clinical evaluation: current status. Adv Drug Deliv Rev 65: 1284-1298.

6. Wei X, Gao J, Zhan C, Xie C, Chai Z, et al. (2015) Liposome-based glioma targeted drug delivery enabled by stable peptide ligands. J Control Release 218: 13-21.

7. Wei X, Zhan C, Shen Q, Fu W, Xie C, et al. (2015) A D-peptide ligand of nicotine acetylcholine receptors for brain-targeted drug delivery. Angew Chem Int Ed Engl 54: 3023-3027.

8. Li C, Shen J, Wei X, Xie C, Lu W (2012) Targeted delivery of a novel palmitylated D-peptide for antiglioblastoma molecular therapy. J Drug Target 20: 264-271.

9. Liu Y, Mei L, Yu Q, Xu C, Qiu Y, et al. (2015) Multifunctional Tandem Peptide Modified Paclitaxel-Loaded Liposomes for the Treatment of Vasculogenic Mimicry and Cancer Stem Cells in Malignant Glioma. ACS Appl Mater Interfaces 7: 16792-16801.

10. Yang Y, Yan Z, Wei D, Zhong J, Liu L, et al. (2013) Tumor-penetrating peptide functionalization enhances the anti-glioblastoma effect of doxorubicin liposomes. Nanotechnology 24: 405101.

11. Yang ZZ, Li JQ, Wang ZZ, Dong DW, Qi XR (2014) Tumor-targeting dual peptides-modified cationic liposomes for delivery of siRNA and docetaxel to gliomas. Biomaterials 35: 5226-5239. 